\title{
PERIODIC BOUNDARY VALUE PROBLEM FOR SECOND ORDER INTEGRO-ORDINARY DIFFERENTIAL EQUATIONS WITH GENERAL KERNEL AND CARATHÉODORY NONLINEARITIES*
}

\author{
JUAN J. NIETO \\ Departamento de Análisis Matemático \\ Facultad de Matemáticas \\ Universidad de Santiago de Compostela \\ SPAIN \\ (Received March 8, 1994 and in revised form November 29, 1994)
}

\begin{abstract}
We study the existence of solutions for the periodic boundary value problem for some second order integro-differential equations with a general kernel. Also we develop the monotone method to approximate the extremal solutions of the problem.
\end{abstract}

KEY WORDS AND PHRASES: Integro-Differential Equation, Upper and Lower Solutions, Carathéodory function

1991 AMS SUBJECT CLASSIFICATION CODES: $45 \mathrm{~J} 05$

\section{INTRODUCTION}

The purpose of this paper is to study the following periodic boundary value problem for a second order nonlinear integro-ordinary differential equation

$$
-u^{\prime \prime}(t)=f(t, u(t), K u(t)), u(0)=u(2 \pi), u^{\prime}(0)=u^{\prime}(2 \pi)
$$

where $f: I \times \mathbf{R}^{2} \rightarrow \mathbf{R}$ is a Carathéodory function, $K$ is an integral operator in $L^{2}(I)$ with kernel $k \in L^{2}(J), J=I \times I$. Related to (1.1) we consider the linear problem

$$
-u^{\prime \prime}(t)+M u(t)+N[K u](t)=h(t), u(0)=u(2 \pi), u^{\prime}(0)=u^{\prime}(2 \pi)
$$

where $M, N \in \mathbf{R}$, and $h \in L^{2}(I)$.

By a solution $u$ of (1.1) we mean a function $u \in H^{2}(I)$ such that the function $t \in I \rightarrow f(t, u(t),[K u](t))$ is a function in $L^{2}(I)$ satisfying the equation for a.e. $t \in I$, and $u(0)=u(2 \pi), u^{\prime}(0)=u^{\prime}(2 \pi)$.

Problem (1.1) is considered in [7] with $f$ continuous and $K$ a Volterra integral operator with positive kernel. The authors developed the monotone iterative method for (1.1) based on a comparison result. As it is pointed out in [6], the method of [7] is not applicable to the general situation. Erbe and Guo studied problem (1.1) with $f$ continuous, and $k$ continuous and positive. They first considered the linear problem (1.2) and gave an estimate on $\|k\|_{\infty}$. We note that in [6], $K u=N T u+N_{1} S u$ with $N, N_{1}$ real numbers, $T$ an integral operator of Volterra type, and $S$ an integral operator of Fredholm type. Problem (1.1) is studied in [8] under the assumption that $f(t, u, v)$ is continuous and increasing in $v$, and in [7] for $f$ continuous and $K$ of Volterra type. Following the ideas of [6] we study (1.1) in the general case, i.e., $f$ is a Carathéodory function and $k$ is an $L^{2}$ kernel. Also, we do not require $k$ to have constant sign on $J$. For the linear problem (1.2) to have a unique solution, we give an estimate on $\|k\|_{2}$ that improves the estimation given in [6], and our estimate is the best possible in the sense that if equality is attained, then the existence-uniqueness result for (1.2) is not valid anymore.

*Research partially supported by DGICYT, Project PB91-0793. 


\section{PBVP FOR SECOND ORDER ORDINARY DIFFERENTIAL EQUATION}

We recall here, for convenience of the reader, some results for the following periodic boundary value problem (PBVP) for a linear second order ordinary differential equation. The problem

$$
-u^{\prime \prime}(t)+M u(t)=h(t) ; \quad u(0)=u(2 \pi) ; \quad u^{\prime}(0)=u^{\prime}(2 \pi)
$$

with $M=m^{2}, m>0$ and $h \in L^{2}(I)$, has a unique solution given by the expression

$$
u(t)=\int_{0}^{2 \pi} G(t, s) h(s) d s,
$$

the Green function $G$ is given by

$$
G(t, s)= \begin{cases}\frac{1}{2 m\left(e^{2 m \pi}-1\right)}\left[e^{m(t-s)}+e^{m(2 \pi-t+s}\right] ; & 0 \leq s \leq t \leq 2 \pi \\ \frac{1}{2 m\left(e^{2 m \pi}-1\right)}\left[e^{m(s-t)}+e^{m(2 \pi-s+t)}\right] ; & 0 \leq t \leq s \leq 2 \pi\end{cases}
$$

Moreover, $G$ is continuous on $J=I \times I$,

$$
\int_{0}^{2 \pi} G(t, s) d s=\frac{1}{M}, \quad \min \{G(t, s):(t, s) \in J\}=\frac{e^{m \pi}}{m\left(e^{2 m \pi}-1\right)}=\mathrm{a},
$$

and $\max \{G(t, s):(t, s) \in J\}=\frac{1+e^{2 m x}}{2 m\left(e^{2 m x}-1\right)} \equiv b$.

Since $G(t, s) \geq$ a $>0$ for every $(t, s) \in J$ we obtain the following maximum principles:

$$
\begin{aligned}
& h \geq 0 \text { a.e. on } I \text { implies } u \geq 0 \text { on } I, \\
& h \leq 0 \text { a.e. on } I \text { implies } u \leq 0 \text { on } I
\end{aligned}
$$

Obviously, $h=0$ implies $u=0$, but if $h>0$ on a set of positive measure of $I$, then $u(t) \geq a \int_{0}^{2 \pi} h(s) d s>0$ for every $t \in i$.

\section{LINEAR INTEGRO-DIFFERENTIAL EQUATIONS}

We now consider the integro-differential problem (1.2) with $M>0$, $N \in \mathbf{R},[K u](t)=\int_{0}^{2 \pi} k(t, s) u(s) d s, k \in L^{2}(J)$ and $h \in L^{2}(I)$.

Note that $K$ is an integral operator, and it could be either of Volterra or Fredholm type. Even $K$ can be of mixed type as in [6] where

$$
K u=N T u+N_{1} S u
$$

with $N, N_{1}$ real numbers, $T$ an integral operator of Volterra type with kernel $k_{0}$, and $S$ an integral operator of Fredholm type with kernel $k_{1}$. Thus, $k=N \cdot k_{0}+N_{1} \cdot k_{1}$.

In what follows, \|\|$_{p}$ denotes the usual norm in $L^{p}, 1 \leq p \leq \infty$.

According to the results of section 2, we have that $u$ is a solution of (1.2) if and only if

$$
u(t)=\int_{0}^{2 \pi} G(t, s)[h(s)-N[K u](s)] d s .
$$

Using Fubini's theorem it is easy to see that for any $t \in I$

$$
-N \int_{0}^{2 \pi} G(t, s)[K u](s) d s=\int_{0}^{2 \pi} \tau(t, s) u(s) d s,
$$

with

$$
\tau(t, s)=-N \int_{0}^{2 \pi} G(t, r) k(r, s) d s
$$

Therefore, equation (3.2) is equivalent to the following abstract equation 


$$
u(t)=w(t)+[T u](t)
$$

where

$$
w(t)=\int_{0}^{2 \pi} G(t, s) h(s) d s
$$

and

$$
[T u](t)=\int_{0}^{2 \pi} \tau(t, s) u(s) d s .
$$

In consequence, $u$ is a solution of the linear problem (1.2) if and only if $u$ is a fixed point of the operator $T_{w}: L^{2}(I) \rightarrow L^{2}(I), T_{w}(u)=w+T u$.

THEOREM 3.1. Suppose that

$$
\|\tau\|_{2}<1
$$

then (1.2) has a unique solution $u=\lim _{n \rightarrow \infty} u_{n}, u_{0} \in L^{2}(I), u_{n+1}=T_{w}\left(u_{n}\right), n \geq 0$. Moreover, the solution is given by the following relations

$$
\begin{gathered}
u(t)=\int_{0}^{2 \pi}[G(t, s)+H(t, s)] h(s) d s, \\
H(t, s)=\int_{0}^{2 \pi} L(t, r) G(r, s) d r, \\
L \equiv \sum_{n=1}^{\infty} \tau_{n}, \quad \tau_{1}=\tau, \quad \tau_{n}(t, s)=\int_{0}^{2 \pi} \tau_{n-1}(t, r) \tau(r, s) d r, \quad n \geq 2 .
\end{gathered}
$$

PROOF. Note that $\left\|T_{w}(u)-T_{w}(v)\right\|_{2} \leq\|\tau\|_{2} \cdot\|u-v\|_{2}$ for any $u, v \in L^{2}(I)$. By the contraction principle of Banach we have that $T_{w}$ has a unique fixed point which is the solution of (1.2). Now, we choose $u_{0}=w$. Using again Fubini's theorem, it is easy to see that

$$
\begin{aligned}
u_{n}(t) & =w(t)+\int_{0}^{2 \pi} H_{n}(t, s) h(s) d s, \\
H_{n}(t, s) & =\int_{0}^{2 \pi} L_{n}(t, r) G(r, s) d r, \\
L_{n} & =\sum_{i=1}^{n} \tau_{i} .
\end{aligned}
$$

We have that $\left\|\tau_{i}\right\|_{2} \leq\|\tau\|_{2}^{i}$, and taking into account (3.5) we see that the series $\sum_{i=1}^{\infty} \tau_{i}$ is convergent in $L^{2}(J)$. We now deduce that $\left\{H_{n}\right\} \rightarrow H$ in $L^{2}(J)$ and the validity of formulas (3.6), (3.7), (3.8).

Taking into account (3.3) we have that $\|\tau\|_{2} \leq\|k\|_{2}\|G\|_{2}$. In consequence, $\|k\|_{2}\|G\|_{2}<1$ implies that the linear problem (1.2) has a unique solution. It is possible to give different estimates for $k$ that imply that (3.5) holds. For instance, if there exists $c>0$ such that

$$
\left|\int_{0}^{2 \pi} k(t, s) d s\right|<c \quad \text { for every } s \in I
$$

then $|\tau(t, s)| \leq b c$ for every $(t, s) \in J$ and $\|\tau\|_{2}<2 \pi b c$. Therefore, $c<\frac{1}{2 \pi b}$ implies that the linear problem (1.2) is uniquely solvable.

In the case that $k \in L^{\infty}(J)$ then $|\tau(t, s)| \leq \frac{|k|_{\infty}}{M}$ for a.e. $(t, s) \in J$. Thus, $\|\tau\|_{2}<\frac{2 \pi|k|_{\infty}}{M}$ and, in this situation, $\|k\|_{\infty}<\frac{M}{2 \pi}$ implies that $\|\tau\|_{2}<1$. 
Note that in the case that $K$ is given by (3.1), Erbe and Guo gave the estimate (see formula (4) in [6])

$$
N_{0}\left\|K_{0}\right\|_{\infty}+N_{1}\left\|k_{1}\right\|_{\infty}<\frac{M}{2 \pi}
$$

which obviously implies (3.5).

A natural question is if Theorem 3.1 remains valid in the critical case $\|\tau\|_{2}=1$. The following example shows that in such a case the linear problem (1.2) may have either no solution or an infinite number of solutions, thus showing that the estimate (3.5) is as sharp as possible.

EXAMPLE. Take $k=c \cdot G, c \in \mathbf{R}$ such that $\|k\|_{2}=1$. The integral operator $T$ associated to $\tau$ is compact and selfadjoint. Thus, +1 or -1 is an eigenvalue of $T$. Suppose that +1 is an eigenvalue and choose $u \neq 0$ with $T u=u$. Thus, by the Fredholm alternative theorem, the equation $u=w+T u$ has either no solution or an infinite number of solutions.

\section{MAXIMUM PRINCIPLE}

We are now interested in obtaining a similar result to (2.4) for the linear integro-differential problem (1.2). Using the representation (3.6) for the solution of (1.2), we see that it is equivalent to show that $G+H \geq 0$ a.e. on $J$. Since $G(t, s) \geq a$ for any $(t, s) \in J$, we can affirm that $G+H \geq 0$ a.e. on $J$ if, for instance, $\|H\|_{\infty} \leq a$. We first give an estimate for $\|H\|_{\infty}$.

THEOREM 4.1. Suppose that $k \in L^{\infty}(J)$ and

$$
\|k\|_{\infty}<\frac{M}{2 \pi} \text {. }
$$

Then, (3.5) holds and

$$
\|H\|_{\infty} \leq \frac{\|k\|_{\infty}}{M\left(M-2 \pi\|k\|_{\infty}\right)} .
$$

PROOF. From the relation (3.3) we deduce that $\tau \in L^{\infty}(J)$, and

$$
\|\tau\|_{\infty} \leq \frac{\|k\|_{\infty}}{M}=d<\frac{1}{2 \pi} .
$$

On the other hand, $\tau_{n} \in L^{\infty}(J)$ for any $n \in N$, and $\|\tau\|_{\infty} \leq d(2 \pi d)^{n-1}$. This implies that the series $\sum_{n-1}^{\infty} \tau_{n}$ is convergent in $L^{\infty}(J)$. Therefore,

$$
\|L\|_{\infty}=\left\|\sum_{n=1}^{\infty} \tau_{n}\right\|_{\infty} \leq \frac{d}{1-2 \pi d}
$$

and

$$
\|H\| \leq \frac{\|L\|_{\infty}}{M} \leq \frac{d}{M(1-2 \pi d)}
$$

which is precisely estimate (4.2).

We note that the right hand side of (4.2) tends to 0 when $\|k\|_{\infty}$ tends to 0 . Thus, we obtain the following maximum principle for the linear equation (1.2).

THEOREM 4.2. Assume that $k \in L^{\infty}(J)$ and

$$
\|k\|_{\infty}<\frac{M m e^{m \pi}}{e^{2 \pi m}-1+2 \pi m e^{\pi m}}=r .
$$

Then, we have that (3.5) holds and $G+H \geq 0$ a.e. on $J$. 
PROOF. We first note that the following inequality holds true:

$$
\frac{m e^{m \pi}}{e^{2 \pi m}-1+2 \pi m e^{\pi m}}<\frac{1}{2 \pi}
$$

Hence, $\|k\|_{\infty}<\frac{M}{2 \pi}$. Now, using (4.2) we have for a.e. $(t, s) \in J$ that

$$
G(t, s)+H(t, s) \geq a-\frac{\|k\|_{\infty}}{M\left(M-2 \pi\|k\|_{\infty}\right)} .
$$

Combining (4.2) and (4.3) we obtain that $\|H\|_{\infty} \leq a$.

Therefore, we can write that $G(t, s)+H(t, s) \geq a-\|H\|_{\infty}>+0$ for a.e. $(t, s) \in J$, completing the proof of the theorem.

As a consequence, we obtain that if inequality (4.3) holds then

$$
h \geq 0(\leq 0) \text { a.e. on } I \text { implies } u \geq 0(\leq 0) \text { a.e. on } J \text {. }
$$

We now consider the case when the kernel has constant $\operatorname{sign}$ on $J$. If $k \leq 0$ a.e. on $J$, then $\tau \geq 0$ a.e. on $J$ and we have that $H \geq 0$ a.e. on $J$. Then, trivially the maximum principle (4.5) holds.

If $k \geq 0$ a.e. on $J$, then $\tau \leq 0$ a.e. on $J$ and the previous reasoning is not valid. However, we have that $(-1)^{n} \tau_{n} \geq 0$ a.e. on $J, n \geq 1$ and this is useful to prove the following result.

THEOREM 4.3. Suppose that $k \in L^{\infty}(J)$ is such that $k \geq 0$ a.e. on $J$ and

$$
\|k\|_{\infty} \leq \frac{m}{8 \pi^{2} e^{m \pi}}\left[\sqrt{\left(e^{2 \pi m}-1\right)^{2}+16 \pi^{2} M e^{2 \pi m}}-\left(e^{2 \pi m}-1\right)\right]=r_{+} .
$$

Then, (3.5) holds and $G+H \geq 0$ a.e. on $J$.

PROOF. We first note that $r_{+}<\frac{M}{2 \pi}$ and then $\|k\|_{\infty}<\frac{M}{2 \pi}$. On the other hand,

$$
L \geq \sum_{\substack{n=1 \\ n \text { odd }}}^{\infty} \tau_{n}
$$

and

$$
\left\|\sum_{n=1}^{\infty} \tau_{n}\right\|_{n \text { odd }} \leq \sum_{\substack{n=1 \\ n \text { odd }}}^{\infty}\left\|\tau_{n}\right\|_{\infty} \leq \sum_{\substack{n=1 \\ n \text { odd }}}^{\infty} d(2 \pi d)^{2 n-2}=\frac{d}{1-(2 \pi d)^{2}}=\frac{M\|k\|_{\infty}}{M^{2}-4 \pi^{2}\|k\|_{\infty}^{2}} .
$$

Hence,

$$
L(t, s) \geq \frac{-M\|k\|_{\infty}}{M^{2}-4 \pi^{2}\|k\|_{\infty}^{2}}
$$

for a.e. $(t, s) \in J$,

$$
H(t, s) \geq \frac{-M\|k\|_{\infty}}{M^{2}-4 \pi^{2}\|k\|_{\infty}^{2}}
$$

for $(t, s) \in J$. In consequence,

$$
G+H \geq a-\frac{-M\|k\|_{\infty}}{M^{2}-4 \pi^{2}\|k\|_{\infty}^{2}}=a_{1}
$$

on $J$. Now, $a_{1} \geq 0$ if and only if

$$
4 \pi^{2} e^{m \pi}\|k\|_{\infty}^{2}+m\left(e^{2 \pi m}-1\right)\|k\|_{\infty}-M^{2} e^{m \pi} \leq 0,
$$

and this is true for $\|k\|_{\infty} \in\left[0, r_{+}\right]$. 


\section{MONOTONE ITERATIVE METHOD}

We now consider the nonlinear equation (1.1). We recall that $f$ is a Carathéodory function if $f(t, \cdot, \cdot)$ is continuous for a.e. $t \in I, f(\cdot, u, v)$ is measurable for any $u, v \in \mathbf{R}$, and for any $R>0$ there exists $\sigma=\sigma_{R} \in L^{2}(I)$ such that $|f(t, u, v)| \leq \sigma(t)$ for a.e. $t \in I$ for any $u, v \in \mathbf{R}$ with $\max (|u|,|v|) \leq R$. Define $H_{2 \pi}^{2}(I)=\left\{u \in H^{2}(I): u(0)=u(2 \pi), u^{\prime}(0)=u^{\prime}(2 \pi)\right\}$. By a solution $u$ of $(1.1)$ we mean a function $u \in H_{2 \pi}^{2}(I)$ such that $t \in I \rightarrow[F(u)](t)=f(t, u(t),[K u](t))$ is a function of $L^{2}(I)$ satisfying the equation for a.e. $t \in I$.

We say that $\alpha \in H_{2 \pi}^{2}(I)$ is a lower solution for (1.1) if $F \alpha \in L^{2}(I)$, and

$$
-\alpha^{\prime \prime}(t) \leq f(t, \alpha(t),[K \alpha](t)) \text { for a.e. } t \in I .
$$

Similarly, we define an upper solution as a function $\beta \in H_{2 \pi}^{2}(I)$ such that $F \beta \in L^{2}(I)$, and

$$
-\beta^{\prime \prime}(t) \leq f(t, \beta(t),[K \beta](t)) \text { for a.e. } t \in I .
$$

In $u \in L^{2}(I)$, in general, $F u$ is not a function of $L^{2}(I)$. The condition that $F$ maps $L^{2}(I)$ into $L^{2}(I)$ is equivalent [1] to the existence of $b \in L^{2}(I), a \in \mathbf{R}$ such that $|f(t, u, v)| \leq b(t)+a(|u|+|v|)$ for a.e. $t \in I$ and every $u, v \in \mathbf{R}$.

Now suppose that condition (3.9) is verified. Obviously, (3.9) is satisfied if, for instance, $k \in L^{\infty}(J)$. However, $k(t, s)=s^{-1 / 2}$ is a kernel that satisfies (3.9) but does not belong to $L^{\infty}(J)$. Then for any $u \in L^{\infty}(I)$ we have that

$$
|[K u](t)|=\left|\int_{0}^{2 \pi} k(t, s) u(s) d s\right|<c\|u\|_{\infty},
$$

and $K u \in L^{\infty}(I)$. In consequence, $|f(t, u,[K u](t))| \leq \sigma_{R}(t)$ for a.e. $t \in I$, where $R=\max \left(\|u\|_{\infty} c\|u\|_{\infty}\right)$, and $F u \in L^{2}(I)$.

Thus, if condition (3.9) holds and $\alpha \in H_{2 \pi}^{2}(I)$, then $K \alpha \in L^{\infty}(I)$ and $F \alpha \in L^{2}(I)$.

If $\alpha, \beta$ are lower and upper solutions of (1.1) respectively, we shall assume that

$$
\alpha \leq \beta \text { on } I \text {. }
$$

Thus, $k \geq 0(\leq 0)$ implies that $K \alpha \leq(z) K \beta$ a.e. on $I$.

In the case that $k \geq 0$ a.e. on $J$, we introduce the following condition: there exist $M>0, N>0$ such that

$$
f(t, u, v)-f(t, w, x) \geq-M(u-w)-N(v-x)
$$

for a.e. $t \in I, \alpha(t) \leq w \leq u \leq \beta(t)$, and $[K \alpha](t) \leq x \leq v \leq[K \beta](t)$.

If $k \leq 0$ a.e. on $J$, we shall use the condition: there exist $M>0, N>0$ such that

$$
f(t, u, v)-f(t, w, x) \geq-M(u-w)-N(x-v) .
$$

for a.e. $t \in I, \alpha(t) \leq w \leq u \leq \beta(t)$, and $[K \beta](t) \leq v \leq x \leq[K \alpha](t)$.

THEOREM 5.1. Assume that $k \in L^{\infty}(J), k \geq 0$ a.e. on $J$, and $\|k\|_{\infty} \leq \frac{r_{+}}{|N|}$. In addition, suppose that there exist $\alpha, \beta \in H_{2 \pi}^{2}(I)$ lower and upper solutions of (1.1) respectively such that (5.3) and (5.4) hold. Then, there exists monotone sequences $\left\{\alpha_{n}\right\} \uparrow \phi$, and $\left\{\beta_{n}\right\} \downarrow \psi$ uniformly on I with $\alpha_{0}=\alpha$ and $\beta_{0}=\beta$. Here $\phi$ and $\psi$ are the minimal and maximal solutions of $(1.1)$ respectively on $[\alpha, \beta]$. Moreover, these sequences verify $\alpha_{0} \leq \ldots \leq \alpha_{n} \leq \ldots \leq \beta_{n} \leq \ldots \leq \beta_{0}$.

PROOF. For $\eta \in[\alpha, \beta]$, let us consider the following linear periodic boundary value problem

$$
-u^{\prime \prime}+M u+N[K u]=h_{\eta}(t), \quad u(0)=u(2 \pi), \quad u^{\prime}(0)=u^{\prime}(2 \pi)
$$


where $h(t)=h_{\eta}(t)=f(t, \eta(t),[K \eta](t))+M \eta+N[K \eta](t)$.

This linear problem has a unique solution $u=A \eta$ in view of Theorem 3.1 since $\|N \cdot k\|_{\infty} \leq r$. Moreover, in this case $G+H \geq 0$ a.e. on $J$.

The operator $A$ is well defined from $[\alpha, \beta]$ to $[\alpha, \beta]$ and $A$ is increasing.

Indeed, let $\eta \in[\alpha, \beta]$ and define $v=u-\alpha$. Thus, using (5.4), we obtain

$$
\begin{gathered}
-v^{\prime \prime}+M v+N K v=f(t, \eta, K \eta)+M \eta+N K \eta-\left(-\alpha^{\prime \prime}+M \alpha+N K \alpha\right) \geq \\
f(t, \eta, K \eta)+M \eta+N K \eta-f(t, \alpha, K \alpha)-M \alpha-N K \alpha \geq 0 .
\end{gathered}
$$

Hence, by virtue of Theorem 4.3 we deduce that $v \geq 0$ on $I$. Similarly, one can show that $u \leq \beta$ on I.

To show the monotonicity of $A$, let $\eta_{i} \in[\alpha, \beta], u_{t}=A \eta_{t}, i=1,2$, and $w=u_{1}-u_{2}$. Hence,

$$
-w^{\prime \prime}+M w+N K w=f\left(t, \eta_{1}, K \eta_{1}\right)+M \eta_{1}+N K \eta_{1}-f\left(t, \eta_{2}, K \eta_{2}\right)-M \eta_{2}-N K \eta_{2} \geq 0
$$

and then, $w \geq 0$.

We now define $\alpha_{0}=\alpha, \alpha_{n+1}=A \alpha_{n}, n \geq 0$. By the properties of the operator $A$, the sequence $\left\{\alpha_{n}\right\}$ is increasing and uniformly bounded on $I$. Then, $\left\{\alpha_{n}\right\} \uparrow \phi$ pointwise on $I$. Writing the integral representation for $A \alpha_{n}$ and using standard arguments we obtain that $\phi$ is actually a solution of (1.1). Analogously, defining $\beta_{0}=\beta, \beta_{n+1}=A \beta_{n}, n \geq 0,\left\{\beta_{n}\right\} \downarrow \psi$, where $\psi$ is solution of (1.1).

To show that $\phi$ and $\psi$ are the minimal and maximal solutions of $(1.1)$ in $[\alpha, \beta]$, let $u \in[\alpha, \beta]$ be a solution of (1.1). Then, $A u=u$, and using the properties of the operator $A$ we have that $\alpha_{n} \leq u \leq \beta_{n}$ for every $n \in N$. Passing to the limit when $n \rightarrow \infty$ we obtain that $\phi \leq u \leq \psi$.

If $k \leq 0$ a.e. on $J$, then we can use directly that $G+H \geq 0$ a.e. on $J$ to obtain the following result.

THEOREM 5.2. Suppose that $k \in L^{\infty}(J), k \leq 0$ a.e. on $J$, and $\|k\|_{\infty} \leq \frac{M}{2 \pi \cdot|N|}$. In addition, assume that there exist $\alpha, \beta \in H_{2 \pi}^{2}(I)$ lower and upper solutions of (1.1) respectively such that (5.3) and (5.5) hold. Then, there exist monotone sequences $\left\{\alpha_{n}\right\} \uparrow \phi$, and $\left\{\beta_{n}\right\} \downarrow \psi$ uniformly on I with

$$
\alpha=\alpha_{0} \leq \ldots \leq \alpha_{n} \leq \ldots \leq \beta_{n} \leq \ldots \leq \beta_{0}=\beta,
$$

and $\phi, \psi$ are the minimal and maximal solutions of (1.1) respectively on $[\alpha, \beta]$.

PROOF. For $\eta \in[\alpha, \beta]$, let us consider the linear problem (5.6). In this case, $K \alpha \geq u=K \eta \geq K \beta$ on $I$. As in the proof of Theorem 5.1 we have that (5.6) has a unique solution $u=A \eta$. The operator $A$ is well defined from $[\alpha, \beta]$ to $[\alpha, \beta]$ and it is increasing. As in the proof of Theorem 5.1 we construct monotone sequences $\left\{\alpha_{n}\right\} \uparrow \phi$ and $\left\{\beta_{n}\right\} \downarrow \psi$, where $\phi$ and $\psi$ are the minimal and maximal solutions of (1.1) respectively between $\alpha$ and $\beta$.

It may occur that

$$
K \alpha \leq K \beta \text { a.e. on } I
$$

even if $\alpha \leq \beta$ and $k$ changes sign on $J$ since $[K \alpha](t)$ depends on the value of $k(t, s) \alpha(s), 0 \leq s \leq 2 \pi$. (For example, take $\varepsilon>0, k(t, s)=1$ for $0 \leq s \leq 2 \pi-\varepsilon, k(t, s)=-1$ for $2 \pi-\varepsilon<s \leq 2 \pi$, and $\alpha=1$, $\beta \equiv 2$.) In such a situation, we can apply the previous results to obtain.

THEOREM 5.3. Suppose that $k \in L^{\infty}(J), N \cdot\|k\|_{\infty}<r$, and (5.3), (5.4), (5.7) hold. Then, there exist monotone sequences $\left\{\alpha_{n}\right\} \uparrow \phi$, and $\left\{\beta_{n}\right\} \downarrow \psi$ uniformly on $I$ with $\alpha_{0}=\alpha, \beta_{0}=\beta$ and $\alpha_{0} \leq \ldots \leq \alpha_{n} \leq \ldots \leq \beta_{n} \leq \ldots \leq \beta_{0}$. Here $\phi$ and $\psi$ are the minimal and maximal solutions of (1.1) respectively on $[\alpha, \beta]$. 
If $K \alpha \geq K \beta$ a.e. on $J$ (even if $k$ has no constant sign on $J$ ), then we have an analogous result using (5.5).

In the general case ( $k$ has no constant sign on $J$ we are able to deal with a linear integral perturbation of the ordinary differential equation $-u^{\prime \prime}=f(t, u)$, being such a perturbation of the type $N \cdot K u, N \in \mathbf{R}$. In concrete, we consider the problem

$$
-u^{\prime \prime}=f(t, u)+N \cdot K u, \quad u(0)=u(2 \pi), \quad u^{\prime}(0)=u^{\prime}(2 \pi) .
$$

We now require the following condition: there exists $M>0$ such that

$$
f(t, u)-f(t, v) \geq-M(u-v)
$$

for a.e. $t \in I, \alpha(t) \leq v \leq u \leq \beta(t)$. In this case, for $\eta \in[\alpha, \beta]$, the linear problem (5.6) reads

$$
-u^{\prime \prime}+M u=f(t, \eta)+M \eta+N K \eta \text {. }
$$

THEOREM 5.4. Consider the nonlinear problem (5.8) were $f$ satisfies condition (5.9). Suppose that $k \in L^{\infty}(I)$ is such that $|N|\|k\|_{\infty}<r$, and that there exist $\alpha, \beta \in H_{2}^{2 \pi}(I)$ lower and upper solution of (5.8) respectively with $\alpha \leq \beta$ on I. Then, there exists monotone sequences $\left\{\alpha_{n}\right\} \uparrow \phi$, and $\left\{\beta_{n}\right\} \downarrow \psi$ uniformly on I with $\alpha=\alpha_{0} \leq \ldots \leq \alpha_{n} \leq \ldots \leq \beta_{n} \leq \ldots \leq \beta_{0}-\beta$. Here $\phi$ and $\psi$ are the minimal and maximal solutions of $(5.8)$ respectively on $[\alpha, \beta]$.

\section{REFERENCES}

[1] APPELL, J. and ZABREIKO, P. P., Nonlinear Superposition Operators, Cambridge University Press, Cambridge, 1990.

[2] BECKER, L. C., BURTON, T. A. and KRISZTIN, T., Floquet theory for a Volterra equation, J. London Math. Soc. (2) 37 (1988), 141-147.

[3] BURTON, T. A., ElOE, P. W. and ISLAM, N. M., Periodic solutions of linear integro-differential equations, Math. Nachr. 147 (1990), 175-184.

[4] BURTON, T. A., ELOE, P. W. and ISLAM, N. M., Nonlinear integro-differential equations and a priori bounds on periodic solutions, Ann. Math. Pura. Appl. IVSer. 161 (1992), 271-283.

[5] CORDUNEANU, C., Integral Equations and Applications, Cambridge University Press, Cambridge, 1990.

[6] ERBE, L. H. and GUO, D., Periodic boundary value problems for second order integrodifferential equations of mixed type, Appl. Anal. 46 (1992), 249-258.

[7] HU, S. and LAKSHMIKANTHAM, V., Periodic boundary value problems for second order integro-differential equations of Volterra type, Appl. Anal. 21 (1986), 199-205.

[8] HU, S. and LEELA, S., Periodic boundary value problem for a second order integro-differential equation of Hammerstein type, Appl. Math. Comput. 25(1988), 219-226.

[9] KAUL, S. K. and VATSALA, A. S., Monotone method for integro-differential equations with periodic boundary conditions, Appl. Anal. 21 (1986), 297-305.

[10] LADDE, G. S., LAKSHMIKANTHAM, V. and VATSALA, A. S., Monotone Iterative Techniques for Nonlinear Differential Equations, Pitman, Boston, 1985.

[11] McKEE, S., Volterra integral and integro-differential equations arising from problems in engineering and science, Bull. Inst. Math. Appl. 24 (1988), 135-138.

[12] NIETO, J. J., Nonlinear second order periodic boundary value problems with Carathéodory functions, Appl. Anal. 34 (1989), 111-128. 


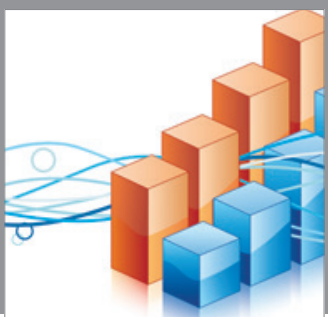

Advances in

Operations Research

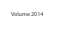

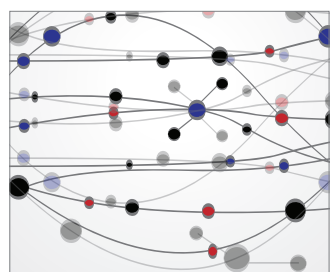

\section{The Scientific} World Journal
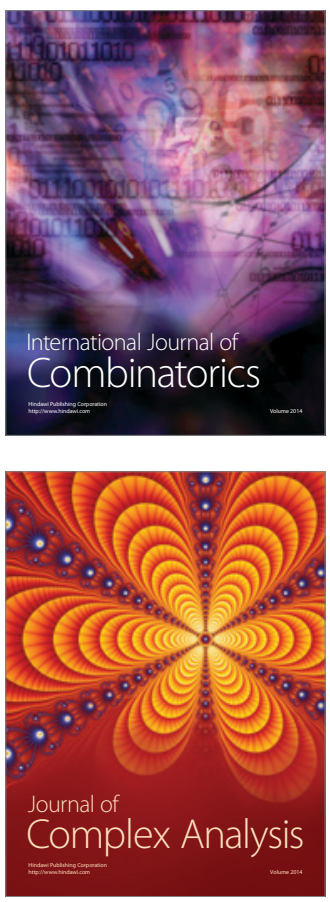

International Journal of

Mathematics and

Mathematical

Sciences
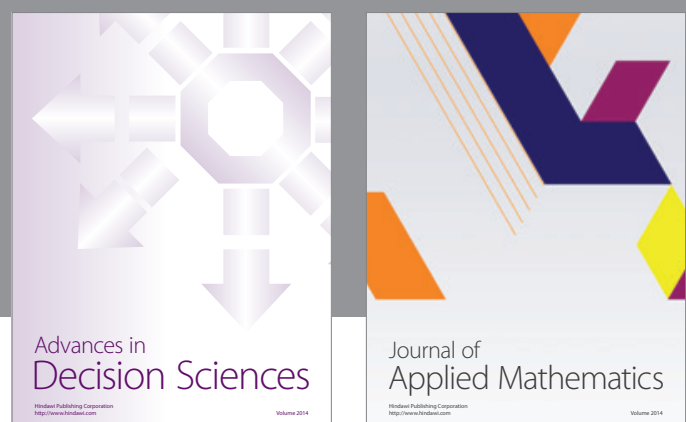

Journal of

Applied Mathematics
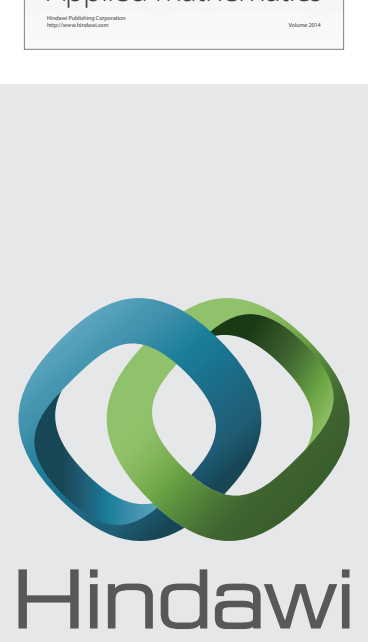

Submit your manuscripts at http://www.hindawi.com
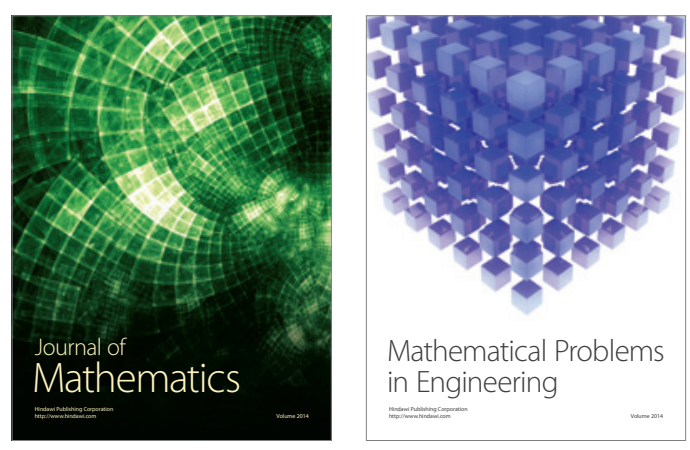

Mathematical Problems in Engineering
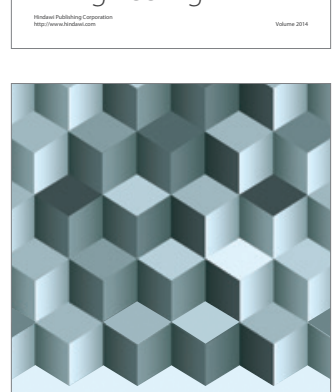

Journal of

Function Spaces
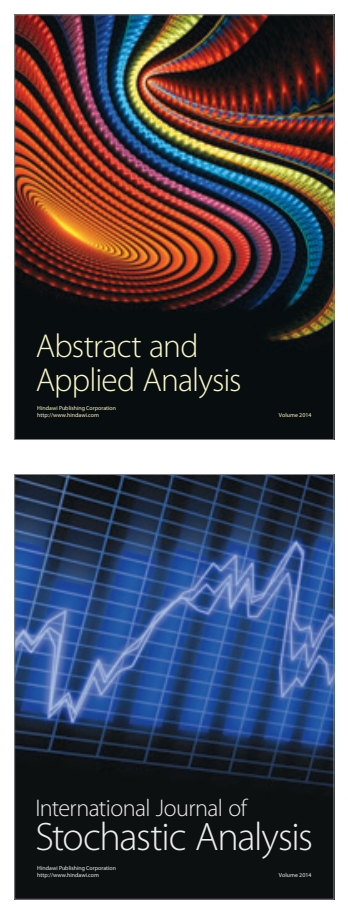

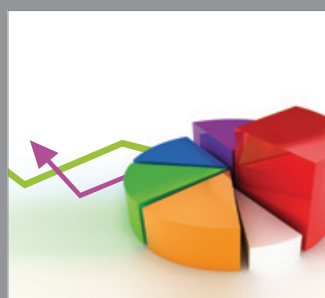

ournal of

Probability and Statistics

Promensencen
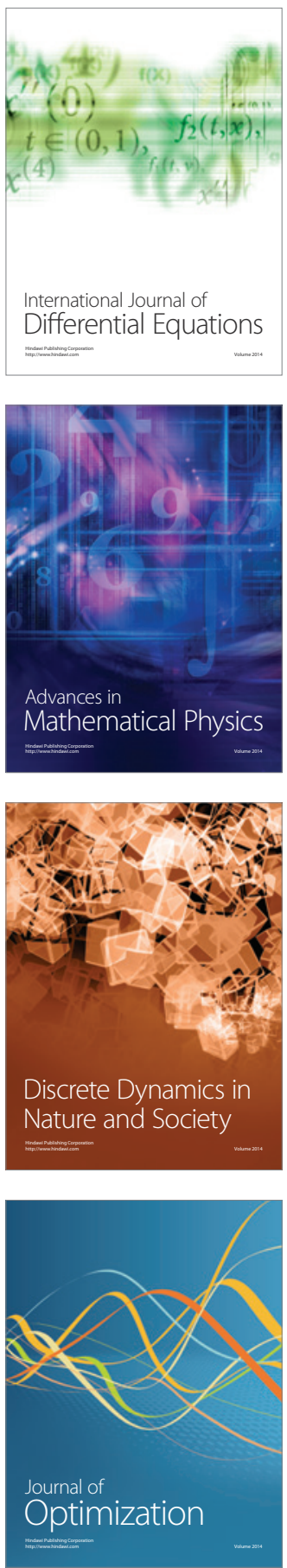